\title{
Cor Triatriatum Dexter: an unusual cause of neonatal cyanosis
}

\section{Cor Triatriatum Dexter: una causa infrecuente de cianosis neonatal}

\author{
Daniel Hurtado-Sierra ${ }^{1 *}$, Oscar Fernández-Gómez', Francisco Manrique-Rincón', \\ Alfonso Buendía-Hernández ${ }^{2}$, and Clara A. Vázquez-Antona ${ }^{3}$
}

\begin{abstract}
${ }^{1}$ Servicio de cardiología pediátrica, Instituto del Corazón de Bucaramanga, Colombia; ${ }^{2}$ Departamento de cardiología pediátrica, Instituto Nacional de Cardiología Ignacio Chávez, Mexico City; ${ }^{3}$ Departamento de ecocardiografía pediátrica, Instituto Nacional de Cardiología Ignacio Chávez, Mexico City, Mexico
\end{abstract}

We present the case of a term newborn, with no significant perinatal history, who was taken to the emergency room at 18 days old for intermittent episodes of cyanosis, with no signs of respiratory distress, oxygen saturation of $85 \%$, arterial gases with moderate hypoxemia, and normal chest X-ray. The transthoracic echocardiogram (TTE) revealed a large undulating membrane that divided the right atrium into two chambers and partially prolapsing through the tricuspid valve (Fig. 1A-D and Video 1). The caval veins drain into the posteromedial chamber, with part of its flow redirected by the membrane to the left atrium through the foramen ovale (Fig. 2A-D and Video 2). Contrast with an agitated saline solution was performed, observing initial accumulation of microbubbles in the posteromedial chamber (Fig. 3A-B).

Cor triatriatum dexter is a rare congenital heart disease $(0.025 \%$ of all congenital heart defects) characterized by the presence of a large membrane that divides the right atrium into two chambers: an posteromedial chamber that receives the flow of the caval veins, and an anterolateral chamber related to the tricuspid valve and right atrial appendage. This membrane represents the persistence of the right valve of sinus venosus, an early embryonic structure that involutes between the $9^{\text {th }}$ and $15^{\text {th }}$ weeks of gestation. The diagnosis can be properly established with a detailed TTE, observing a large linear echogenic structure that divides the right atrium into two chambers, with insertion to the roof of the atrium (crista terminalis) and systemic venous flow partial obstruction. The differential diagnosis must be established with the Chiari network, which has the echocardiographic appearance of a floating network of fibers, with multiple insertions in the upper part of the right atrium, and with the eustachian valve located at the orifice of the inferior vena cava, with little mobility ${ }^{1-3}$.

\section{Funding}

This research has received no specific grant from public agencies, commercial, or non-profit.

\section{Conflicts of interest}

None.

\section{Ethical disclosures}

Protection of human and animal subjects. The authors declare that no experiments were performed on humans or animals for this study.
Available online: $12-11-2020$ Arch Cardiol Mex (Eng). 2021;91(3):327-329 www.archivoscardiologia.com 2604-7063 / @ 2020 Instituto Nacional de Cardiología Ignacio Chávez. Published by Permanyer. This is an open access article under the CC BY-NC-ND license (http://creativecommons.org/licenses/by-nc-nd/4.0/). 


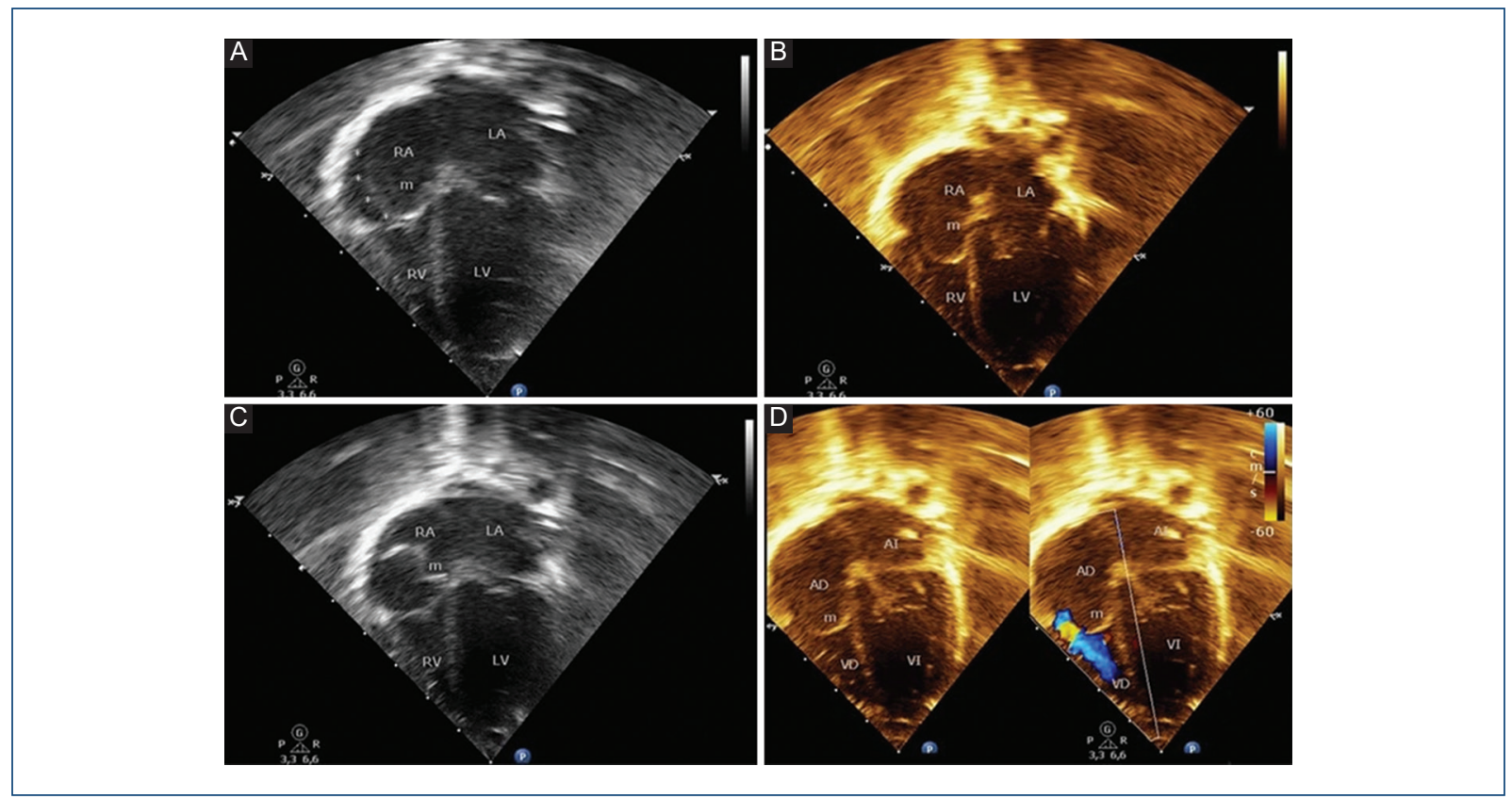

Figure 1. Apical four-chamber transthoracic echocardiographic images. A-C: Illustrating a membrane (m) that divides the right atrium into two chambers and partially prolapsing through the tricuspid valve. D: Echocardiographic image with color Doppler shows eccentric flow through the tricuspid valve from the outer part of the right atrium to right ventricle (with no significant gradient). RA: right atrium; LA: left atrium; RV: right ventricle; LV: left ventricle; M: membrane; FO: foramen ovale; SVC: Superior vena cava.

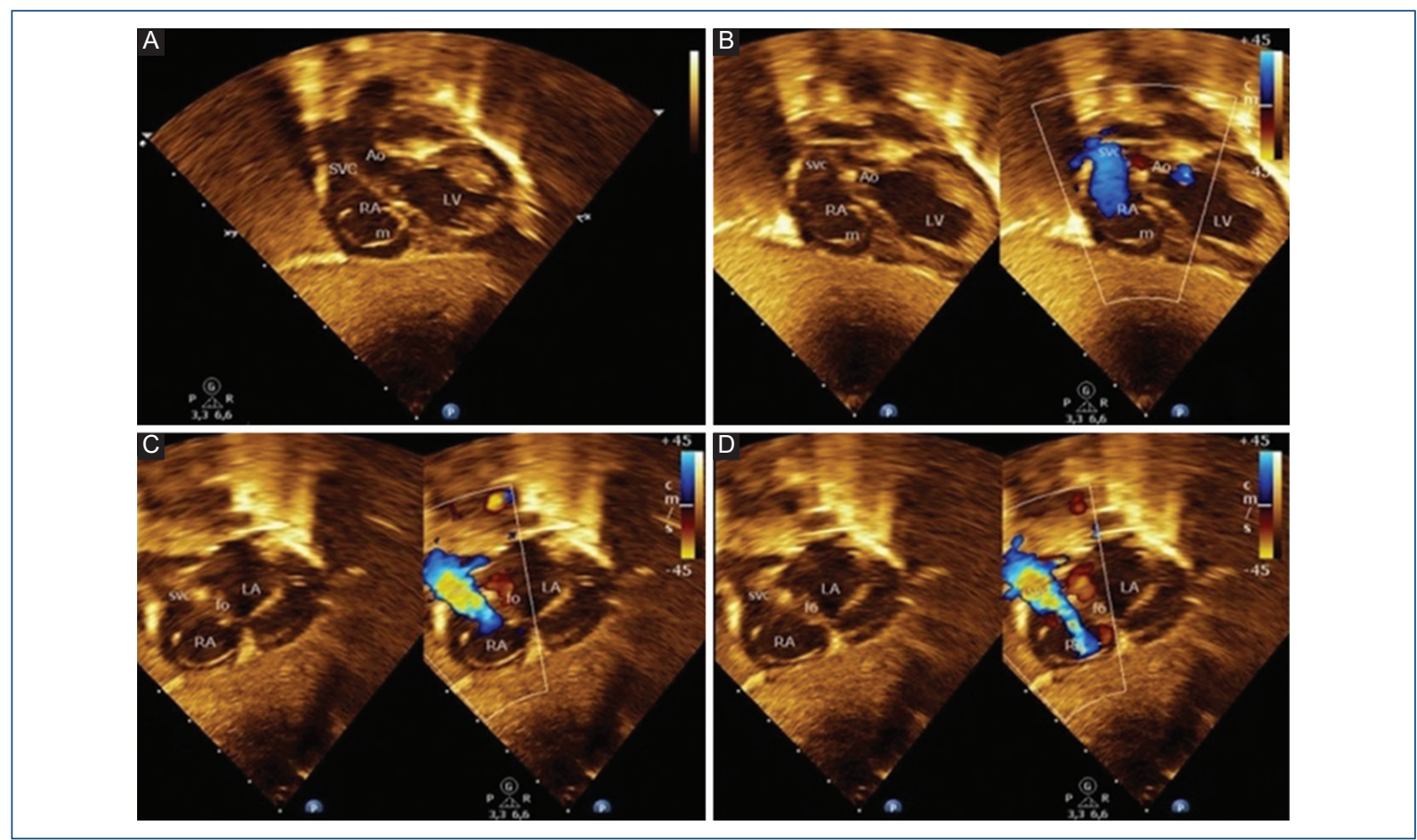

Figure 2. A: Two-dimensional and B-D: Two-dimensional color Flow Doppler echocardiography images from the subcostal window. A: The Cor-Triatriatum Dexter membrane $(\mathrm{m})$ that separates the right atrium into two chambers. B-D: The inner part of right atrium receives the systemic venous blood from superior vena cava which is directed by the Cor-triatriatum membrane to the left atrium through the foramen ovale. 


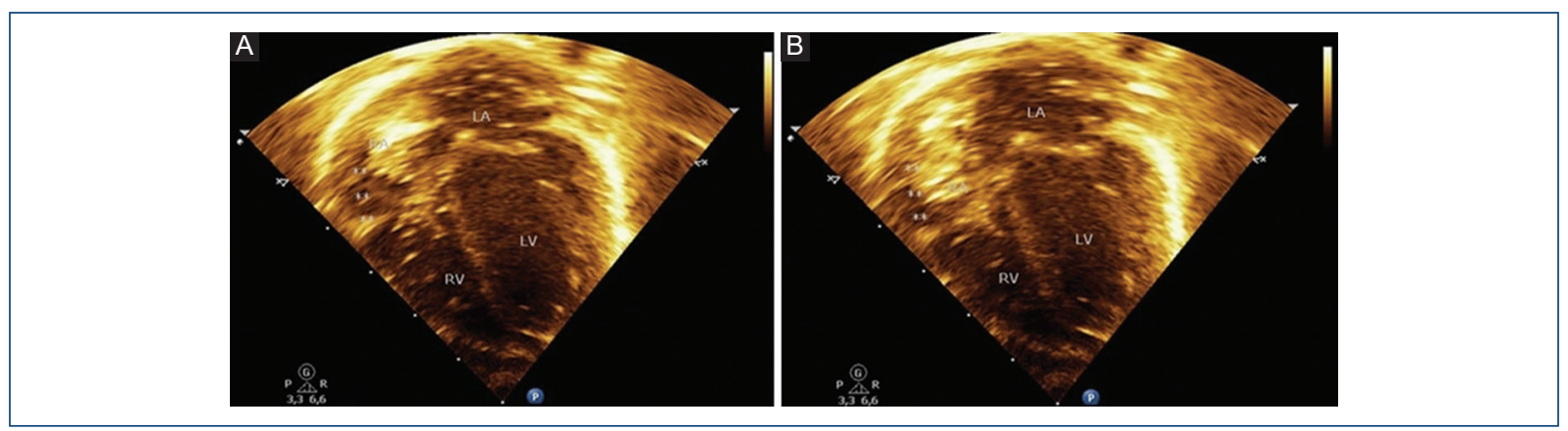

Figure 3. A-B: Contrast echocardiography showing the initial accumulation of microbubbles in the posteromedial chamber.

Confidentiality of data. The authors declare that they have followed the protocols of their work center on the publication of patient data.

Right to privacy and informed consent. The authors declare that no patient data appear in this article.

\section{References}

1. Alghamdi $\mathrm{MH}$. Cor triatriatum dexter: a rare cause of cyanosis during neonatal period. Ann Pediatr Cardiol. 2016;9:46-8.

2. Galli MA, Galletti L, Schena F, Salvini L, Mosca F, Danzi GB. A rare case of neonatal cyanosis due cor triatriatum dexter and a review of the literature. J Cardiovasc Med (Hagerstown). 2009;10:535-8.

3. Moral S, Ballesteros E, Huguet M, Panaro A, Palet J, Evangelista A. Differential diagnosis and clinical implications of remnants of the right valve of the sinus venosus. J Am Soc Echocardiogr. 2016;29:183-94. 